\title{
DIFUSIÓN Y COMPARABILIDAD DE LA INFORMACIÓN ECONÓMICO-FINANCIERA ON-LINE: EL CASO DE IBEROAMÉRICA*
}

\author{
JOSÉ ANTONIO PEÑA RAMOS**, CÉSAR VARGAS DÍAZ*** \& IVÁN MEDINA IBORRA**** \\ UNIVERSIDAD DE GRANADA (ESPANAA) - UNIVERSIDAD PABLO DE OLAVIDE (SEVILLA- ESPAÑA)
}

Recibido/ Received/ Recebido: 10/01/2012 - Aceptado/ Accepted/Aprovado: 15/03/2012

\begin{abstract}
Resumen
El objetivo del siguiente trabajo es generar un ranking a partir de los datos consultados del 2009 de los Ministerios de Economía y Hacienda o en su caso de Finanzas de los países de Iberoamérica más transparentes en divulgar información económico-financiera a nivel on-line. Para ello, se propone como propuesta metodológica un cuestionario e índices de transparencia de la divulgación de información en el entorno económico y financiero. Entre los principales hallazgos, podemos mencionar que los Ministerios de México, España y Chile salieron como los mejores valorados, sin embargo, Panamá y Honduras fueron los menos transparentes en divulgar dicha información

Palabras clave: e-gobierno, índices, información, transparencia económica y financiera.

\section{DISCLOSURE AND COMPARABILITY OF THE ON-LINE ECONOMIC-FINANCIAL INFORMATION: IBEROAMERICA CASE}

\begin{abstract}
The objective of this paper is to create a ranking from the on-line economic-financial data reviewed in Ministries of economy and finance, or just finance depending on the name used in iberoamerican countries, of 2009. For this purpose, a methodological proposal is made, which includes a questionnaire and indexes of information disclosure in economic and financial environment. Among the main results, Ministries of Mexico, Spain and Chile are the best ranked of all, however, Panama and Honduras were the least transparent countries in disclosure such information.
\end{abstract}

Keywords: e-government, indexes, information, economic and financial transparence.

* El presente artículo se inserta en una de las principales líneas de investigación del Instituto de e-Gobierno, de naturaleza interuniversitaria, con sedes en España, México y Bolivia.

** Doctor en Ciencia Política. Área de Ciencia Política y de la Administración. Departamento de Derecho Público. Facultad de Derecho. Universidad Pablo de Olavide (Sevilla - España). Correo electrónico: japer@upo.es

*** Doctor en Ciencias Económicas. Departamento de Economía Internacional y de España. Facultad de Ciencias Económicas y Empresariales. Universidad de Granada. Correo electrónico: cvargasd@ugr.es

**** Doctor en Ciencia Política. Departamento de Ciencia Política y Derecho Público. Facultad de Ciencias Políticas y Sociología. Universidad Autónoma de Barcelona. Correo electrónico: ivan.medina.iborra@gmail.com 


\title{
DIFUSÃO E COMPARABILIDADE DA INFORMAÇÃO ECONÔMICO-FINANCEIRA ON-LINE: O CASO DE IBERO-AMÉRICA
}

\section{Resumo}

\begin{abstract}
O objetivo do seguinte trabalho é criar um ranking a partir de dados conferidos em 2009 dos Ministérios de Economia, Fazenda ou Finanças, dos países mais transparentes em divulgar informação econômico-financeira a nível on-line, de Iberoamérica. Para isso, propõe-se como metodologia um questionário e índices da transparência da divulgação de informação no meio econômico e financeiro. Entre as principais conclusões, podemos mencionar que os Ministérios de México, Espanha e Chile foram os mais bem avaliados. No entanto, Panamá e Honduras foram os menos transparentes em divulgar referida informação.
\end{abstract}

Palavras chave: e-Governo, índices, informação, transparência econômica e financeira.

Vargas, C. Peña, J. \& Medina, I. (2012) Difusión y comparabilidad de la Información Económicofinanciera on-line: El Caso de Iberoamérica. En: Revista de la Facultad de Ciencias Económicas de la Universidad Militar Nueva Granada. rev.fac.cienc.econ, XX (1)

JEL: $M 39$.

\section{Introducción}

Las Tecnologías de la Información y la Comunicación (TIC) y, especialmente, Internet, son hoy un instrumento para que el ciudadano pueda acceder a la información económico-financiera y no financiera relativa a la gestión de los recursos del Gobierno. Al mismo tiempo, ambas son también un vehículo esencial para que las entidades públicas lleven a cabo la rendición de cuentas al ciudadano, teniendo en cuenta, además, las recientes reformas para adoptar modelos de gestión pública, considerando como ejemplo las experiencias de las entidades privadas.

Es así que los organismos internacionales como la Unión Europea (UE), Fondo Monetario Internacional (FMI), Organización para la Cooperación y el Desarrollo Económico (OCDE) y Banco Mundial (BM), entre otros más, y los Gobiernos Nacionales de cada país, han puesto en marcha iniciativas de e-Gobierno y su medición ha permitido conocer la existencia de diferentes niveles de desarrollo dependiendo de países, regiones, ciudades y pueblos, es decir, en todos sus ámbitos. Este es también un aspecto de especial trascendencia, puesto que sus ventajas serán reales en función del grado de desarrollo alcanzado.
En este contexto, las motivaciones de nuestra investigación fueron la creciente importancia de las TIC en las entidades públicas, así como las contribuciones que Internet puede desempeñar en el modelo de divulgación de información pública al detectar la escasez de estudios sobre este tema y los beneficios que genera tanto en los países desarrollados como en desarrollo.

El objetivo que perseguimos es dotar a las entidades públicas de una guía que facilite la transparencia en la divulgación de información económico-financiera pública on-line o e-información, sobre su actuación y satisfacer las expectativas de los ciudadanos-clientes sobre el conocimiento de la actuación pública. Frente a la inclusión de información incompleta y dispersa, el seguimiento de las recomendaciones que formulamos tiene carácter integrador. Además provee a las entidades públicas de un instrumento para incrementar su nivel de responsabilidad y transparencia, lo que redundará en su legitimación social.

Así pues, el objetivo fundamental es analizar en qué medida y de qué manera se divulga la información económico-financiera hacia una mayor transparencia y rendición de cuentas. Para ello, planteamos como propuesta un cuestionario e índices de la 
transparencia de la divulgación de información en el entorno económico y financiero, con el objetivo de crear un ranking de los ministerios más transparentes en divulgar dicha información. Presentamos el estudio para los Ministerios de Economía y Hacienda o, en su caso, de Finanzas de los países iberoamericanos y el caso de Colombia. Con respecto a Colombia es importante considerar la investigación de Cano (2010), la cual presentara las principales experiencias de adopción, adaptación o de armonización de las Normas Internacionales de Contabilidad, que han tenido algunos de los principales países latinoamericanos, tales como Argentina, Brasil, Bolivia, Chile, Costa Rica, Ecuador, Perú, y Venezuela, con el fin de asimilarlos como experiencias exitosas para la dinámica globalizadora en la cual debe integrarse Colombia si no quiere permanecer al margen de los procesos de internacionalización económica que se viven en el mundo desde hace ya más de treinta años.

Por último, también mencionar, que se ha incluido en particular a España con el fin de comparar el nivel de divulgación en ambos escenarios.

\section{Construcción del cuestionario de la e-información}

Desde el punto de vista metodológico, se ha procedido a la elaboración del diseño de un cuestionario basado en el documento publicado principalmente por AECA (2002) y otros como Alt \& Lassen (2005a, 2005b), como también FASB (2000, 2001, 2002), IASC (1999), Spaul (1997) y Trites (1999), para el ámbito de empresa y negocios; así mismo, en los trabajos previos realizados por Vargas (2009a, 2009b) y Caba et al. (2002), López et al. (2002), en el ámbito público; y también en los informes de organismos internacionales (OCDE, 2004; FMI, 1999). Todos ellos servirán para captar aquellos datos o variables consideradas relevantes para el estudio, con el fin de que cualquier potencial usuario de Internet pudiera formarse una opinión fundada acerca de la gestión económica y financiera realizada por la entidad pública a través de una página Web.

Así, nuestro interés no se centra sólo en el análisis del tipo de información financiera, sino también en la información económica que difunden los organismos públicos a través de sus páginas Web, que se denominará información económico-financiera o einformación. Hay que señalar que el estudio vale la pena por la presencia de determinadas cualidades que la información económico-financiera divulgada debe cumplir, como el contenido, las características, formatos e interacción, y el esfuerzo que los gobiernos públicos han realizado para la construcción de una página Web que facilite al usuario su consulta.

Se ha pretendido mostrar los aspectos más básicos de la información económico-financiera, para contrastar la divulgación en las páginas Web de los organismos públicos. Es así, que los agregados adoptados para el análisis de cada uno de estos siete bloques (véase Tabla 1), se clasificaron en el cuestionario mediante una lista de 42 variables informativas que se denominan Ui. Estas variables son dicotómicas, toman los valores 1 y 0 , según el organismo público incluya o no la información económico-financiera estudiada en su sitio Web. Las variables que estudian los Formatos de Presentación han sido ponderadas con un menor peso que las demás, pues se estudia solamente la presencia o no de determinados formatos. La Tabla 1 resume todas las variables a estudiar, agrupadas en cuatro grandes bloques y alguno de ellos, a su vez, dividido en más bloques. Como se ha comentado, existen 42 variables que hay que consultar en cada página Web a estudiar, dándole el valor 0 ó 1 con su correspondiente ponderación a cada una, de manera que el máximo de valoración total es de 40 puntos.

Las variables han sido agrupadas en los siguientes grupos de primer y segundo nivel, que pasamos a comentar de manera individualizada:

\subsection{Contenido informativo (CI)}

Está formado por un total de 22 variables que estudian la información económica, socio-económica y financiera que presentan las páginas web estudiadas y, a su vez, están agrupadas en cuatro grupos:

- Información económica (IE): Contiene 6 variables que deben ser difundidas en los organismos públicos nacionales, ya que se trata de variables 
macroeconómicas, como son el IPC, la inflación, la inversión pública, la inversión extranjera directa, el PIB y el PNB. Cabe mencionar que si se hace un análisis en el ámbito regional y local es probable que estas variables no se difundan, aunque entendemos que debería de existir esta información en las Web en todos los ámbitos o por lo menos, poder acceder a esta información mediante vínculos, ya que se trata de una información relevante para cualquier ciudadano.

- Información Socio-Económica (ISE): Contiene 5 variables, concretamente los datos de población, de actividad económica, de trabajo, de renta, consumo y si existe un plan estratégico.

- Información Financiera de Carácter Presupuestario (IFP): Formado por un total de 6 variables, entre las que se han incluido tanto información sobre el presupuesto aprobado como sobre la liquidación del mismo y sobre el cuadro de financiación representada por el flujo de tesorería.

- Información de Carácter Económico-Financiera (IEF): Incluye 5 variables con información referente a deuda viva a largo plazo y su variación, activos fijos no financieros y su variación, y la cuenta de resultados.

\subsection{Características del contenido: Característi- cas de la Información Económico-Financiera (CIC)}

Está formada por 12 variables y se centra en el carácter de la información de los informes técnicos, su comparabilidad, comprensibilidad, fiabilidad, periodicidad y relevancia de la información contable (tabla 1).

\subsection{Formatos de presentación Electrónicos (FE)}

Está formado por 6 variables que estudian la presencia o no de seis formatos concretos en la información suministrada por la página Web estudiada. Los formatos electrónicos más adecuados en los que podría soportar la información económico-financiera, por la posibilidad que otorga al usuario, para poder manipular, cambiar o resumir la información públi- ca adaptándolo a sus necesidades, son las hojas de cálculo Excel (preferiblemente el formato .xls) o los documentos de texto de Word y Power Point, o en el lenguaje XML, que está adquiriendo una gran relevancia en el ámbito de las empresas para la transmisión de la información financiera (AECA, 2002). Estos formatos podrían ser acompañados por otros que, aunque sean de carácter descargable, o bien no permiten tal manipulación como es Pdf, o simplemente son de texto como el HTML. La valoración de los mismos será 1 para PDF, 0,75 para XML y HTML y 0,5 para Word, Excel y Power Point.

\subsection{Interacción con el Usuario: Solicitar Información (SI)}

Es importante para los organismos públicos interactuar con el ciudadano o usuario, para lo cual es necesario que existan solicitudes y formularios para rellenar y poder ser enviados por medios digitales, con el fin de minimizar coste en tiempo y dinero. Está formado por 2 variables, una que estudia posibilidad de realizar una solicitud a través de un formulario y otra a través de e-mail.

Para poder medir estas variables se elabora un índice de transparencia de información, el cual medirá la información económico-financiera que divulgan los gobiernos a través de sus páginas Web.

\section{Construcción de los índices de la e-información}

Una vez sean revisadas y analizadas las páginas Web de cada organismo público ya sea este a nivel nacional, regional y local, siguiendo estrictamente los controles comentados en el apartado anterior. Como ya se ha indicado, dichas variables solamente pueden tomar los valores 1 (si se difunde la información relativa a la variable) y 0 (si no se difunde en la página Web). Concretando, se define la variable Ui (j) de la siguiente manera: $\mathrm{Ui}(\mathrm{j})=1$, si la característica que define a la variable (i) está presente por el organismo público $(\mathrm{j})$ y Ui $(\mathrm{j})=0$, si no está presente; por tanto, Ui (j) para i entre 1 y n y jentre 1 y m.

- $\quad$ El Índice de Divulgación de la Información Total del Gobierno (DITG): se define como la califi- 
Tabla 1. Construcción del cuestionario de divulgación de información ${ }^{1}$

\begin{tabular}{|c|c|c|c|}
\hline Grupos & Código & Variable & Variación \\
\hline \multirow{19}{*}{$\begin{array}{l}\text { (1) Contenido } \\
\text { informativo }\end{array}$} & \multicolumn{2}{|r|}{ Información económica (IE) } & 6 \\
\hline & U2 & Inflación & 1 \\
\hline & U4 & Inversión extranjera directa & 1 \\
\hline & U5 & Producto Interno Bruto (PIB) & 1 \\
\hline & U6 & Producto Nacional Bruto (PNB) & 1 \\
\hline & \multicolumn{2}{|r|}{ Información socioeconómica (ISE) } & 5 \\
\hline & U8 & Datos de actividad económica & 1 \\
\hline & U9 & Datos de trabajo & 1 \\
\hline & U10 & Datos de renta y consumo & 1 \\
\hline & U11 & Plan estratégico & 1 \\
\hline & \multicolumn{2}{|c|}{ Información financiera de carácter presupuestario (IFP) } & 6 \\
\hline & U12 & Información presupuesto aprobado & 1 \\
\hline & \multicolumn{2}{|c|}{ Información financiera de carácter económico-financiero (IEF) } & 5 \\
\hline & U18 & Deuda viva a largo plazo & 1 \\
\hline & U19 & Variación de la deuda viva & 1 \\
\hline & $\mathrm{U} 20$ & Activos fijos no financieros & 1 \\
\hline & U21 & Variación de activos & 1 \\
\hline & $\mathrm{U} 22$ & Cuenta de resultados económicos & 1 \\
\hline & \multicolumn{2}{|r|}{ Características de la información } & 12 \\
\hline \multirow{5}{*}{$\begin{array}{l}\text { (II) } \\
\text { Características } \\
\text { del contenido }\end{array}$} & U23 & Carácter completo: Presupuestaria & 1 \\
\hline & U24 & Carácter completo: Financiera & 1 \\
\hline & U25 & Comparabilidad: Resúmenes comparativos & 1 \\
\hline & U26 & Comparabilidad: Datos de ejercicios anteriores & 1 \\
\hline & U27 & Comparabilidad: Datos de entidades similares & 1 \\
\hline \multirow{7}{*}{$\begin{array}{l}\text { (III) Formatos de } \\
\text { presentación }\end{array}$} & U35 & PDF & 1 \\
\hline & U36 & HTML & 0,75 \\
\hline & U37 & IML & 0,75 \\
\hline & U38 & Excel & 0,50 \\
\hline & U39 & Power Point & 0,50 \\
\hline & U20 & Word & 0,50 \\
\hline & \multicolumn{2}{|r|}{ Solicitar información } & 2 \\
\hline \multirow{2}{*}{$\begin{array}{l}\text { (IV) Interacción } \\
\text { con el usuario }\end{array}$} & $\mathrm{U} 41$ & Solicitud a través de formulario & 1 \\
\hline & U42 & Solicitud a través de email & 1 \\
\hline & & & 40 \\
\hline
\end{tabular}

cación total obtenida por una entidad pública, después de la valoración de las 42 variables informativas (Ui) vistas en el apartado anterior, con respecto a la Puntuación Máxima Posible (PMPA).

- $\quad$ El Índice de Divulgación de la Información Total de cada Variable (DITV): se define como la calificación total obtenida por la correspondiente variable (Ui), después de la valoración de la misma en la totalidad de las entidades públicas integran- tes de la muestra, con respecto a la Puntuación Máxima Posible de cada Variable (PMPV).

La Puntuación Máxima Posible (PMP): viene dada por la calificación que habría obtenido la página Web de la entidad pública si cada variable adoptase el valor más alto, en nuestro caso 40 puntos (ver Tabla 1 ), mientras que la puntuación máxima posible por variable (PMPV) en caso de que se analice el nivel de divulgación de una variable en el colectivo de entidades publi-

1 Fuente: Elaboración propia. 
cas analizadas, vendría dado por la calificación que habría obtenido la referida variable si se reflejase en las páginas Web de toda la muestra véase los siguientes índices:

$$
\begin{gathered}
D I T G^{(j)}=\sum_{i=1}^{n} U_{i}^{(j)} / G \\
D I T V_{(i)}=\sum_{i=1}^{m} U_{i}^{(j)} / V
\end{gathered}
$$

donde:

Ui : Variables informativas

m: Número de la muestra de las variables

$n$ : Número de la muestra de entidades públicas

$V$ : Número total de variables en nuestro caso 42

G: Número total de la muestra (gobiernos públicos)

Así mismo, la Divulgación de la Información Total de cada Variable dependiente (IE, ISE, IFP, IEF, CIC, FE y SI) dependerá de las correspondientes variables independientes (Ui).

Por ejemplo, para el caso de ISE:

$$
\begin{gathered}
D I T V_{I S E}=\frac{1}{5} \sum_{I}^{5} \operatorname{DITV}_{(i)} \\
=\frac{1}{5} \sum_{i=1}^{5}\left\{\sum_{j=1}^{n} U_{i}^{(j)} / P M P V\right\}
\end{gathered}
$$

Sobre la base propuesta en la construcción del cuestionario, podremos obtener el análisis discriminatorio para las cuatro secciones: el contenido informativo, características informativas, el formato de presentación y la interacción con el ciudadano. Para realizar el análisis de grupos, podemos determinar en qué medida, cada una de las variables dependientes obedece de las citadas variables independientes, de igual manera, podemos ver las ecuaciones para cada una de ellas.

$$
\begin{aligned}
& \text { Cont. Inf. }=\sum_{i=1}^{k} X_{i j} / n^{o} \\
& \text { Caract. Inf. }=\sum_{i=1}^{k} \Xi_{i \varphi} / n^{o} \\
& \text { Form. Prest. }=\sum_{i=1}^{k} X_{i j} / n^{o} \\
& \text { Int.Usu. }=\sum_{i=1}^{k} X_{i j} / n^{o}
\end{aligned}
$$

donde:

$\mathrm{X}_{\mathrm{ij}}$ : representa las variables que establecen la divulgación económico-financiera on-line n: es el número de variables por grupo

Conviene hacer notar que para la construcción en los índices se asume la sustituibilidad total entre todas las variables y se agrega por media aritmética simple. Por lo tanto, nace el Índice de Transparencia de divulgación información Económico-Financiera on-line (e-ITEF) que se puede resumir en la siguiente ecuación:

$$
e-I T E F=\sum_{i=1}^{k}(I E+I S E+I F P+I E F+C I C+F E+S I)
$$

\section{Aplicación al Ámbito Latinoamericano}

Una vez construido el cuestionario y creados los índices, todo ello enfocado hacia una transparencia y responsabilidad a través de la divulgación de la información económico-financiera pública online, se pasa a analizar el nivel de divulgación de información en un caso concreto: Iberoamérica.

\section{1. Ámbito de investigación}

Cabe mencionar que el análisis y metodología a utilizar podría aplicarse en cualquier tipo de entidad pública, ya sea gobierno central, comunidades autónomas, gobiernos regionales y corporaciones locales, etc. En nuestro caso, por diversos motivos, nos vamos a centrar en los gobiernos a nivel central. El primero de esos motivos se encuentra en el papel activo de las Administraciones Centrales y, en particular, de los Ministerios encargados de llevar la 
parte económica y financiera que, como agentes al servicio de la sociedad en su conjunto, han experimentado una evolución notablemente creciente en los últimos años, tal como se pudo evidenciar en la políticas públicas sobre e-Gobiernos a nivel latinoamericano.

Además, las exigencias de la ciudadanía a través de las tecnologías, lo que se conoce como democracia electrónica e-Democracia, sometida a una fuerte presión fiscal y mayor transparencia ha cambiado la mentalidad más reivindicativa que en anteriores décadas, centrándose sobre todo, en el manejo de los recursos económicos -minimizar la corrupción-, es otro motivo, para ver el desarrollo que vienen ejerciendo los e-Gobiernos iberoamericanos a través de sus Ministerios y como éstos servirán de ejemplo y de modelo a los diferentes ámbitos públicos regional, provincial y local.

Así las cosas, el objetivo consiste en analizar las páginas Web de los gobiernos, a través de sus respectivos Ministerios de Economía y Hacienda o en su caso de Finanzas, para evaluar el grado de transparencia, de difusión de información económico-financiera que se suministra al ciudadano.

En la Tabla 2 se recoge la composición de la muestra examinada, clasificada por países y sus respectivos ministerios.

La selección de estos ministerios nos garantiza cierta homogeneidad y comparabilidad de la información. En primer lugar, en competencias y obligaciones, ya que una gran mayoría de ellos cuentan con un marco normativo general, este sea constitucional, legal a través de reglamento o decretos presidenciales para difundir información y a la vez rendir cuentas al ciudadano, además de aplicar políticas públicas sobre e-Gobierno en diferentes ámbitos. La revisión de los contenidos de las páginas Web de los ministerios integrantes de la muestra fue realizada a partir del mes de septiembre de 2007 hasta septiembre de 2009, por lo que no se han tenido en cuenta posibles modificaciones posteriores de tales contenidos.

\subsection{Análisis de los resultados}

Se recogen los índices de transparencia de la divulgación de información económico-financiera que muestran el nivel de divulgación de las páginas Web de los ministerios integrantes de la muestra. El nivel de divulgación de información económico-financiera más alto lo alcanza el Ministerio de México que muestra información relevante para todas las variables informativas seleccionadas, seguido del Ministerio de España con $(0,98)$, Chile $(0,92)$ y Brasil $(0,90)$.

Seguidamente, los Ministerios de Argentina, Costa Rica, Guatemala, Paraguay, Bolivia y Nicaragua con una media por encima de los $(0,80)$ y por debajo de estos los Ministerios de Colombia, Uruguay, Jamaica, Perú y Venezuela los cuales presentan un nivel de divulgación de información entorno a $(0,70)$, seguido de los Ministerios de Salvador $(0,68)$, Ecuador $(0,66)$ y Cuba $(0,62)$, y por último, presentan índices de información inferiores o iguales a $(0,50)$, esto se debe principalmente por recoger escasas variables informativas, entre estos están los Ministerios Honduras, Panamá y Republica Dominicana.

El mayor índice medio de divulgación de información económico-financiera para el caso Centro América es el Ministerio Costa Rica $(0,86)$ y en el caso de Sur América el Ministerio de Chile $(0,92)$. Por su parte, el menor índice medio de información económico-financiera se obtiene para el Ministerio de Panamá y República Dominicana.

En cuanto al nivel de divulgación de las variables informativas seleccionadas en el cuestionario, reflejadas en el Tabla 4, se analizan dichas variables por separado para mayor claridad.

\subsubsection{Información económica}

Entre estas variables las más divulgadas por los ministerios son el Producto Interno Bruto (PIB) y la Inversión Pública con un $22 \%$, seguidas por la Inflación $(20 \%)$ y Inversión extranjera directa (19\%), y entre las que menos información suministran los Ministerios están el Índice Precios al Consumidor $(15 \%)$ y Producto Nacional Bruto (PNB) con un $2 \%$ (véase Tabla 5). 
Tabla 2. Ministerios analizados²

\begin{tabular}{|c|c|c|c|}
\hline Países & Ministerios & $\begin{array}{c}\text { Índice } \\
\text { divulgación }\end{array}$ & Ranking \\
\hline Argentina & Ministerio de Economía de Argentina & 0,88 & 5 \\
\hline Bolivia & Ministerio de Hacienda de Bolivia & 0,85 & 9 \\
\hline Brasil & Ministerio de Hacienda de Brasil & 0,90 & 4 \\
\hline Chile & Ministerio de Economía y Energía de Chile & 0,92 & 3 \\
\hline Colombia & Ministerio de Hacienda y Crédito Público de Colombia & 0,74 & 11 \\
\hline Costa Rica & Ministerio de Hacienda de Costa Rica & 0,86 & 6 \\
\hline Cuba & Ministerio de Finanzas y Precios de Cuba & 0,62 & 13 \\
\hline Ecuador & Ministerio de Economía y Finanzas de Ecuador & 0,66 & 17 \\
\hline España & Ministerio de Hacienda de España & 0,98 & 2 \\
\hline Guatemala & Ministerio de Economía de Guatemala & 0,86 & 7 \\
\hline Honduras & Ministerio de Hacienda y Economía de Honduras & 0,59 & 19 \\
\hline Jamaica & Ministerio de Hacienda y Finanzas de Jamaica & 0,74 & 13 \\
\hline México & Ministerio de Hacienda y Crédito Público de México & 1,00 & 1 \\
\hline Nicaragua & Ministerio de Hacienda y Crédito Público de Nicaragua & 0,81 & 10 \\
\hline Panamá & Ministerio de Economía y Finanzas de Panamá & 0,55 & 20 \\
\hline Paraguay & Ministerio de Hacienda de Paraguay & 0,86 & 8 \\
\hline Perú & Ministerio de Economía y Finanzas de Perú & 0,72 & 14 \\
\hline República Dominicana & $\begin{array}{l}\text { Ministerio de Hacienda y Economía de República Do- } \\
\text { minicana }\end{array}$ & 0,52 & 21 \\
\hline El Salvador & Ministerio de Hacienda de El Salvador & 0,68 & 16 \\
\hline Uruguay & Ministerio de Economía y Finanzas de Uruguay & 0,74 & 12 \\
\hline Venezuela & Ministerio de Finanzas de Venezuela & 0,71 & 15 \\
\hline & & \multicolumn{2}{|c|}{$\begin{array}{c}\text { Promedio: 0,77 } \\
\text { Máximo: 1,00 } \\
\text { Mínimo: 0,52 } \\
\text { Muestra: } 21\end{array}$} \\
\hline
\end{tabular}

2 Fuente: Elaboración propia. 
Tabla 3. Nivel de divulgación de la información económico-financiera por ministerios ${ }^{3}$

\begin{tabular}{|c|c|c|c|}
\hline Países & Ministerios & $\begin{array}{c}\text { Índice } \\
\text { divulgación }\end{array}$ & Ranking \\
\hline Argentina & Ministerio de Economía de Argentina & 0,88 & 5 \\
\hline Bolivia & Ministerio de Hacienda de Bolivia & 0,85 & 9 \\
\hline Brasil & Ministerio de Hacienda de Brasil & 0,90 & 4 \\
\hline Chile & Ministerio de Economía y Energía de Chile & 0,92 & 3 \\
\hline Colombia & Ministerio de Hacienda y Crédito Público de Colombia & 0,74 & 11 \\
\hline Costa Rica & Ministerio de Hacienda de Costa Rica & 0,86 & 6 \\
\hline Cuba & Ministerio de Finanzas y Precios de Cuba & 0,62 & 13 \\
\hline Ecuador & Ministerio de Economía y Finanzas de Ecuador & 0,66 & 17 \\
\hline España & Ministerio de Hacienda de España & 0,98 & 2 \\
\hline Guatemala & Ministerio de Economía de Guatemala & 0,86 & 7 \\
\hline Honduras & Ministerio de Hacienda y Economía de Honduras & 0,59 & 19 \\
\hline Jamaica & Ministerio de Hacienda y Finanzas de Jamaica & 0,74 & 13 \\
\hline México & Ministerio de Hacienda y Crédito Público de México & 1,00 & 1 \\
\hline Nicaragua & Ministerio de Hacienda y Crédito Público de Nicaragua & 0,81 & 10 \\
\hline Panamá & Ministerio de Economía y Finanzas de Panamá & 0,55 & 20 \\
\hline Paraguay & Ministerio de Hacienda de Paraguay & 0,86 & 8 \\
\hline Perú & Ministerio de Economía y Finanzas de Perú & 0,72 & 14 \\
\hline República Dominicana & $\begin{array}{l}\text { Ministerio de Hacienda y Economía de República Do- } \\
\text { minicana }\end{array}$ & 0,52 & 21 \\
\hline El Salvador & Ministerio de Hacienda de El Salvador & 0,68 & 16 \\
\hline Uruguay & Ministerio de Economía y Finanzas de Uruguay & 0,74 & 12 \\
\hline Venezuela & Ministerio de Finanzas de Venezuela & 0,71 & 15 \\
\hline & & \multicolumn{2}{|c|}{$\begin{array}{c}\text { Promedio: 0,77 } \\
\text { Máximo: 1,00 } \\
\text { Mínimo: 0,52 } \\
\text { Muestra: } 21\end{array}$} \\
\hline
\end{tabular}

3 Fuente: Elaboración propia. 
Tabla 4. Nivel de divulgación por variables de información ${ }^{4}$

\begin{tabular}{|c|c|c|}
\hline Código & Variable & Índice divulgación \\
\hline \multicolumn{2}{|r|}{ Información económica } & 0,75 \\
\hline U1 & Índice Precios al Consumidor (IPC) & 0,67 \\
\hline U2 & Inflación & 0,95 \\
\hline U3 & Inversión Pública & 0,95 \\
\hline U4 & Inversión extranjera directa & 0,86 \\
\hline U5 & Producto Interno Bruto (PIB) & 1,00 \\
\hline U6 & Producto Nacional Bruto (PNB) & 0,10 \\
\hline \multicolumn{2}{|r|}{ Información socioeconómica } & 0,77 \\
\hline U1 & Datos de población & 0,95 \\
\hline $\mathrm{U} 2$ & Datos de actividad económica & 0,95 \\
\hline U3 & Datos de trabajo & 0,19 \\
\hline $\mathrm{U} 4$ & Datos de renta y consumo & 0,76 \\
\hline U5 & Plan estratégico & 1,00 \\
\hline \multicolumn{2}{|r|}{ Información financiera de carácter presupuestario } & 0,56 \\
\hline U1 & Información presupuesto aprobado & 1,00 \\
\hline U2 & Gastos ejecutados & 0,24 \\
\hline U3 & Ingresos ejecutados & 0,29 \\
\hline U4 & Modificaciones presupuestarias & 0,29 \\
\hline U5 & Superávit o déficit & 1,00 \\
\hline U6 & Flujos de tesorería (cuadro de financiación) & 0,52 \\
\hline \multicolumn{2}{|c|}{ Información financiera de carácter económico-financiero } & $\mathbf{0 , 5 8}$ \\
\hline U1 & Deuda viva a largo plazo & 0,95 \\
\hline $\mathrm{U} 2$ & Variación de la deuda viva & 0,57 \\
\hline U3 & Activos fijos no financieros & 0,29 \\
\hline $\mathrm{U} 4$ & Variación de activos & 0,24 \\
\hline U5 & Cuenta de resultados económicos & 0,86 \\
\hline \multicolumn{2}{|r|}{ Características de la información } & 0,67 \\
\hline $\mathrm{U} 1$ & Carácter completo: Presupuestaria & 1,00 \\
\hline U2 & Carácter completo: Financiera & 0,43 \\
\hline U3 & Comparabilidad: Resúmenes comparativos & 0,57 \\
\hline U4 & Comparabilidad: Datos de ejercicios anteriores & 0,71 \\
\hline U5 & Comparabilidad: Datos de entidades similares & 0,19 \\
\hline U6 & Comprersibilidad: Incluye ratios y gráficos & 0,52 \\
\hline U7 & Comprersibilidad: Comentarios explicativos & 0,95 \\
\hline U8 & Fabilidad: Verifica la información oficial & 0,95 \\
\hline U9 & Oportunidad: Periocidad de la información suministrada & 0,86 \\
\hline U10 & Oportunidad: Por lo menos dos idiomas & 0,00 \\
\hline U11 & Revelancia: Informes técnicos elaborados por el propio & 0,95 \\
\hline $\mathrm{U} 12$ & Revelancia: Información segregada & 0,90 \\
\hline \multicolumn{2}{|r|}{ Formatos electrónicos } & 0,62 \\
\hline U1 & PDF & 1,00 \\
\hline $\mathrm{U} 2$ & HTML & 0,57 \\
\hline U3 & IML & 0,25 \\
\hline U4 & Excel & 0,24 \\
\hline U5 & Power Point & 0,21 \\
\hline U6 & Word & 0,21 \\
\hline \multicolumn{2}{|r|}{ Solicitar información } & 0,74 \\
\hline U1 & Solicitud a través de formulario & 0,62 \\
\hline $\mathrm{U} 2$ & Solicitud a través de email & 0,86 \\
\hline
\end{tabular}

4 Fuente: Elaboración propia. 
Tabla 5. Porcentaje de divulgación de información económica ${ }^{5}$

\begin{tabular}{|l|l|}
\hline \multicolumn{1}{|c|}{ Información económica } & $\%$ \\
\hline Índice de Precios al Consumidor (IPC) & 15 \\
\hline Inversión Pública & 22 \\
\hline Producto Interno Bruto (PIB) & 22 \\
\hline Inflación & 20 \\
\hline Inversión extranjera directa & 19 \\
\hline Producto Nacional Bruto (PNB) & 2 \\
\hline
\end{tabular}

\subsubsection{Información socio-económica}

Dentro de las variables referentes a la información socio-económica, las que más divulgación tienen son la población (26\%) y el plan estratégico $(25 \%)$, es decir, son las variables suministradas por los ministerios. El suministro de información sobre la actividad económica es de $24 \%$ (agrícola y ganadera; comercial; financiera e industrial). Esta última ocupa el tercer lugar, mientras renta y consumo (renta disponible; indicadores de consumo) es ofrecida por un porcentaje similar de ministerios $(20 \%)$ y los datos de la situación trabajo (tasa de paro registrado; situación laboral y profesional de la población activa) tan solo un $5 \%$ (ver tabla 6 ).

Tabla 6. Porcentaje de divulgación de información socioeconómica ${ }^{6}$

\begin{tabular}{|l|c|}
\hline \multicolumn{1}{|c|}{ Información socio-económica } & $\%$ \\
\hline Datos de población & 26 \\
\hline Datos de trabajo & 5 \\
\hline Plan estratégico & 25 \\
\hline Datos de actividad económica & 24 \\
\hline Datos de renta y consumo & 20 \\
\hline
\end{tabular}

\subsubsection{Información financiera de carácter presupuestario}

La información que se ofrece de carácter presupuestario y de tesorería, se observa en la Tabla 7. Algo más del $99 \%$ de los ministerios suelen presentar información sobre el presupuesto aprobado. Por otro lado, se observa la ausencia de información sobre el presupuesto ejecutado, pues únicamente 5 de los 21 ministerios analizados (20\%) presentan información sobre los gastos e ingresos ejecutados y un $25 \%$ de estos ministerios lo hacen del resultado presupuestario. De este modo, se ve que existe un alto consenso en cuanto a su inclusión en la página web del ministerio de información sobre el presupuesto aprobado, pero no sobre la ejecución del mismo.

Tabla 7. Porcentaje de divulgación de información financiera de carácter presupuestario ${ }^{7}$

\begin{tabular}{|l|c|}
\hline \multicolumn{1}{|c|}{$\begin{array}{c}\text { Información financiera de } \\
\text { carácter presupuestario }\end{array}$} & $\%$ \\
\hline Información presupuesto aprobado & 30 \\
\hline Gastos ejecutados & 6 \\
\hline Ingresos ejecutados & 8 \\
\hline Modificaciones presupuestarias & 9 \\
\hline Superávit o déficit & 30 \\
\hline Flujos de tesorería (cuadro de financiación) & 17 \\
\hline
\end{tabular}

Se otorga una escasa importancia a la divulgación de información en Internet sobre las modificaciones realizadas al presupuesto, pues el $9 \%$ de las páginas Web de los ministerios analizados carecen de esta información. Los niveles de importancia atribuidos a suministrar información sobre flujos de tesorería también son bajos (sólo se presenta en el $17 \%$ de las páginas Web de los ministerios analizados).

Fuente: Elaboración propia.

Fuente: Elaboración propia.

Fuente: Elaboración propia. 


\subsubsection{Información financiera de carácter económico-financiera}

Respecto a la difusión por Internet de información sobre posición financiera como se puede apreciar en la Tabla 8, la situación prácticamente es la misma que para el suministro de información sobre presupuesto ejecutado. De hecho, más del $60 \%$ de los ministerios presentan una información financiero-patrimonial deficiente, pues la información suministrada sobre los ítems considerados es la siguiente:

La divulgación en Internet de información sobre deuda viva de largo plazo es suministrada tan solo por un $32 \%$ de las páginas Web de los ministerios objeto de estudio, bajando este porcentaje cuando nos centramos en variación de deuda viva al $20 \%$.

Tabla 8. Porcentaje de divulgación de información financiera de carácter económico-financiero8

\begin{tabular}{|l|c|}
\hline \multicolumn{1}{|c|}{$\begin{array}{c}\text { Información financiera de carácter } \\
\text { económico-financiero }\end{array}$} & $\%$ \\
\hline Deuda viva a largo plazo & 32 \\
\hline Activos fijos no financieros & 10 \\
\hline Cuenta de resultados económicos & 30 \\
\hline Variaciones de la deuda viva & 20 \\
\hline Variación de activos & 8 \\
\hline
\end{tabular}

La información referida a activos fijos no financieros también es baja (10\%), sin embargo la cuenta de resultados económicos es más elevada alcanzando $30 \%$, ya que únicamente seis ministerios ofrecen la posibilidad de conocer los activos no financieros con los que cuenta la entidad, la variación que éstos han sufrido durante un periodo de tiempo y el resultado económico-financiero obtenido.

En síntesis, para la muestra de ministerios analizados en este trabajo, el grado de implantación de Internet como medio de divulgación de la información presupuestaria y financiero-patrimonial pública está siendo muy bajo, optándose en todo caso por informar sobre el presupuesto aprobado y no el ejecutado. No debemos olvidar, que todos los ítems de este bloque de información analizados se deben elaborar y suministrar obligatoriamente por los ministerios según la legislación actualmente vigente.

\subsubsection{Características de la información financiera pública}

Para este bloque se recogen datos vinculados con el grado de cumplimiento de determinadas características de la información financiera pública. Para ello se han incluido ítems que revisan la presencia de aquellas cualidades que, según la literatura existente en materia de reportes digitales, destacan por su importancia en la divulgación de la información financiera. En este orden de cosas, nos hemos centrado en el estudio de la presencia de: i) carácter completo de la información presupuestaria y contable; ii) comprensibilidad o claridad; iii) comparabilidad; iv) relevancia y v) fiabilidad. Del análisis ya mencionado podemos extraerse las siguientes consideraciones (ver ilustración 1).

En relación al carácter completo de la información, como ya se señalaba se distinguía entre la posibilidad de que un usuario potencial de la información financiera pública tuviera acceso a un informe amplio de la información financiero-patrimonial y de la información presupuestaria o bien únicamente a un resumen. Así, nos encontramos que casi el total de los ministerios suministran información presupuestaria en Internet, cuelgan en su Web un informe bastante completo, no siendo así, la parte financiera a pesar que existe un gran avance al respecto (45\%).

En cuanto a la comparabilidad de la información económico-financiera on-line, hemos observado, en primer lugar, que el $70 \%$ de las Web permiten a sus usuarios disponer de información financiera pública de más de dos ejercicios económicos para poder elaborar sus propios informes comparativos. En segundo lugar, son menos las entidades públicas que optan por realizar dichos informes comparativos y los cuelgan en la red concretamente el $60 \%$.

El análisis de la comprensibilidad nos lleva a centrarnos, en primer lugar, en la posibilidad existente

8 Fuente: Elaboración propia. 
en las Web de encontrarnos ratios o gráficos financieros que nos faciliten la comprensión de dicha información. Así, el 55\% de estas Web consideran conveniente la inclusión de esta opción y respecto a mostrar los comentarios a dicha información (95\%).

Continuando nuestro análisis con la característica denominada relevancia, observamos, primeramente, que todos los ministerios han colgado en la red sus informes técnicos. En segundo lugar, destaca el hecho de la posibilidad que existe en el $90 \%$ de las páginas de consultar la información de forma segregada, es decir, por sectores o actividades. Sin embargo, este comentario no debe de confundirnos dado que como la información que suministran, la mayoría de ellos es sobre el presupuesto aprobado, y este suele ser bastante completo, es lógico que se muestre información segregada sobre los diferentes organismos públicos y empresas que componen el ministerio. Señalar, que ningún ministerio presenta por lo menos dos idiomas como oportunidad para consultar toda la información por el usuario, pero si existen algunos ministerios que dan la posibilidad de consultar en otro idioma, pero no toda la información.

Por último, al analizar la fiabilidad de la información financiera hemos observado que también es significativa, esto se debe, a la importancia que tiene esta información para los otros ámbitos del sector público como de la ciudadanía.

\subsubsection{Formatos electrónicos}

Respecto a los formatos electrónicos, podemos deducir que los ministerios divulgan su información principalmente en formato $p d f$, también utilizan el formato $\mathrm{html}$ pero en menor grado, y los formatos menos utilizados son XML Excel, Word y Power Point, que tan solo divulgan su información los ministerios de México, Argentina, Chile y España.

\subsubsection{Solicitar la información}

Los ministerios para interactuar con el ciudadano utilizan el correo electrónico, para hacer consultas y a través de este se puede solicitar información, casi un $80 \%$ de los ministerios utiliza este método, de igual manera vienen utilizando solicitudes electrónicas para descargar y en su caso rellenar en la misma solicitud, pero este método solo lo utiliza el $50 \%$ aproximadamente.

En cuanto al nivel de divulgación de las variables informativas seleccionadas, reflejadas en la Tabla 9, el mayor índice medio de la transparencia de información, es la información económica, información

Ilustración 1. Características de la información contable ${ }^{9}$

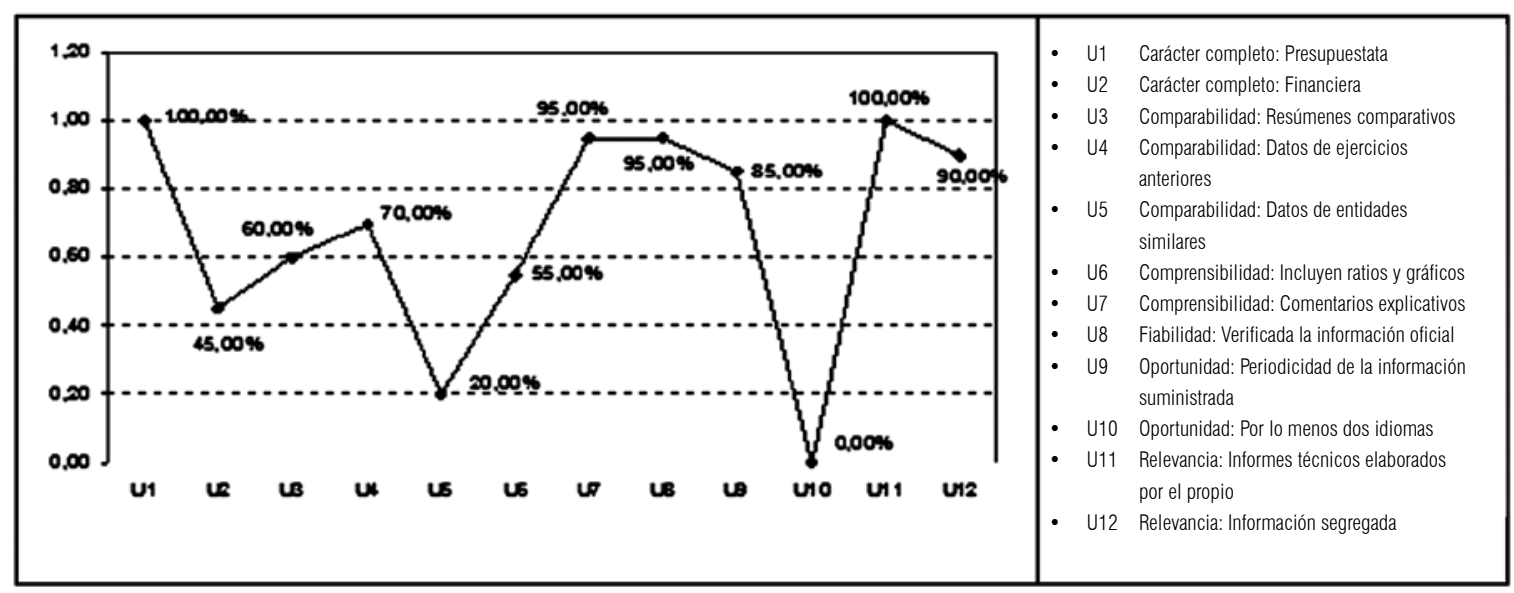

9 Fuente: Elaboración propia. 
socio-económica y la interacción con el ciudadano alcanzando el $16 \%$ respecto del total de variables analizadas, seguida por las características de la información contable con un $14 \%$, la información económico-financiera y los formatos electrónicos con un $13 \%$ y, por último, la información con carácter presupuestario con el $12 \%$.

Tabla 9. Nivel de divulgación por variables ${ }^{10}$

\begin{tabular}{|l|c|}
\hline \multicolumn{1}{|c|}{ Variables } & $\%$ \\
\hline Información económica & 16 \\
\hline Información financiera de carácter presupuestario & 12 \\
\hline Características de la información & 14 \\
\hline Información socio-económica & 16 \\
\hline Información financiera de carácter económico financiero & 13 \\
\hline Formatos electrónicos & 13 \\
\hline Solicitar información & 16 \\
\hline
\end{tabular}

El aumento del nivel de divulgación de las variables que se encuentran por encima del $50 \%$, se puede explicar por la relevancia ó el interés que muestran los e-gobiernos iberoamericanos a través de sus ministerios en difundir datos económico-financieros esenciales para los ciudadanos y que es una responsabilidad contraída por dichos gestores públicos hacia la transparencia de e-información.

Es así, que lo analizado muestra que el índice de la transparencia de la información económico-financiera genera resultados alentadores y se podría decir significativos, si se desea alcanzar los objetivos de potenciar la transparencia en la gestión y la rendición de cuentas a nivel financiero y económico, pero resulta imprescindible contar con un marco normativo no solo a nivel nacional sino en todos sus niveles en el ámbito público, para generar la obligación legal que los gobiernos deben rendir cuentas a los ciudadanos, para demostrar la responsabilidad contraída por los gobernantes y gestores ante los votantes que los han elegido.

\section{Conclusiones}

A juicio de los investigadores, se ponen de manifiesto que las administraciones públicas analizadas se están potenciando, aunque se encuentran aún en un momento incipiente. Por eso existe un elevado grado de heterogeneidad que caracteriza el momento actual.

El análisis descriptivo llevado a cabo en un primer momento, muestra el elevado número de Ministerios que de manera voluntaria ponen sus estados financieros a disposición del ciudadano a través de Internet. En algunos países están encaminando ha normalizar todo el tema de transparencia y divulgación de información, pero es evidente que aun queda mucho recorrido en el tema de normas y por su puesto de hacerlas cumplir.

El documento más difundido por estas administraciones es el presupuesto del Estado, incluido en el sitio Web de los ministerios analizados. Son varios los ministerios que divulgan el presupuesto completo de varios ejercicios económicos, siendo esta variable la más difundida por los ministerios.

El resto de los documentos que se han pretendido localizar en la red, cuentas anuales de obligada elaboración, informes, memorias o indicadores de gestión, anuarios o informes anuales, han podido ser consultados en un número de ministerios realmente mayores a la media. A pesar que solo el $45 \%$ acompaña la información financiera con comentarios, la divulgación de información segregada y la utilización de ratios y gráficos son dos cualidades que también se presentan aproximadamente en un $50 \%$ de las páginas Web revisadas. Los formatos electrónicos más utilizados para la presentación de los datos son los tradicionales pdf y html, pero señalar que están empezando a difundir información en otros formatos como XML, Excel, Power Point y Word. El medio de interacción más extendido consiste en poner a disposición del usuario la dirección de correo electrónico de un responsable financiero.

10 Fuente: Elaboración propia. 
Un análisis comparativo de los distintos ministerios analizados nos lleva a destacar los de mayor grado de cumplimiento de la e-información. Ésta está concentrada básicamente en los ministerios de México y España, que presentan un alto índice de divulgación de información no sólo presupuestaria y socioeconómica, sino también de información financiera económica. Con respecto al resto de países, especialmente en Colombia, existe un gran desarrollo en estos años del e-Gobierno, si bien queda camino por recorrer hacia la transparencia de la divulgación de información económica y financiera.

Con respecto a las futuras líneas de investigación, los resultados obtenidos en este ámbito dan una perspectiva amplia para abordar futuras líneas de investigación tales como: códigos de buenas prácticas para el de diseño de las páginas Web, código de buenas prácticas para la transparencia informativa, creación de nuevas leyes y normas que regulen y cooperen con una mayor transparencia de la información y la rendición de cuentas, no solo en el ámbito nacional sino internacional, estudios entorno a la transparencia y la responsabilidad corporativa y a la comparabilidad (bechnmarking) de la información. Por último, mencionar que la metodología desarrollada del cuestionario e índices, es extendible y mejorable a cualquier ámbito público, sea nacional, regional, provincial y local, etc.

\section{Referencias}

Alt, J. \& Lassen, D. (2005a) The political budget cycle is where you can't see it: transparency and fiscal Manipulation. Universidad de Copenhague, EPRU Working Paper No 3.

Alt, J. \& Lassen, D. (2005b) Fiscal transparency, political parties, and debt in OECD countries. En: European Economic Review, 50: $1403-1439$.

Asociación Española de Contabilidad y Administración de Empresas (AECA) (2002) "Código de Buenas Prácticas para la Divulgación de Información Financiera en Internet". En: Documento de Nuevas Tecnologías y Contabilidad, 1.

Caba, C.; López, A. M. \& Rodríguez, M. P. (2002) "La divulgación de la información financiera del sector público nacional y provincial argentino en la era digital". En: Enfoques. Contabilidad y Administración, 4: 78-93.

Cano Morales, A. M. (2010) "Algunas Experiencias Exitosas en Latinoamérica Relacionadas con la Estandarización Contable". En: Revista Facultad de Ciencias Económicas: Investigación y Reflexión, Universidad Militar Nueva Granada, XVIII (2): 207-226.

Financial Accounting Styards Board (FASB) (2000). Electronic distribution of business reporting information. Business Reporting Research Project. FASB.

Financial Accounting Styards Board (FASB) (2001). Improving business reporting: insights into enhancing voluntary disclosures. Steering committee report. Business Reporting Research Projet. Financial Accounting Styards Board. FASB.

Financial Accounting Styards Board (FASB) (2002). Update of electronic distribution of business reporting information. Survey of business reporting research information on companies' internet sites. Business Reporting Research Project, FASB.

Fondo Monetario Internacional (FMI) (1999). Manual de Transparencia Fiscal. Fondo Monetario Internacional.

International Accounting Styars Committe (IASC) (1999). Business Reporting on the Internet. Discussion Paper. Londres: IASC.

López, A. M.; Caba, C. \& Rodríguez, M. P. (2002) "El impacto de las nuevas tecnologías en la difusión de información financiera pública de los países de la Asociación Interamericana de Integración (ALADI)". En: Revista Interamericana de Contabilidad, 11: 19-32.

Organización para la Cooperación y el Desarrollo Económico (OCDE) (2004). "E-government studies: the e-government imperative". Disponible en: http://www1.oecd.org/publications/ebook/4203071e.pdf

Spaul, B. (1997). Corporate dialogue in the digital age. Londres: The Institute of Chartered Accountants in England and Wales (ICAEW).

Trites, G. (1999). The Impact of Technology on Financial and Business Reporting. Canadian Institute of Chartered Accountants.

Vargas, C. (2009a) "Transparencia de la información económicofinanciera a través del e-Gobierno o Gobierno Electrónico: Caso Español". En: Perspectiva, 24: 59-90.

Vargas, C. (2009b). "E-Gobierno: hacia una gestión ágil transparente al servicio del ciudadano". Disponible en: http://ayuntamientodeogijares.es/opencms/export/sites/default/ogijares/modules/ descargas/periodico/periodico_ene_2009_3.pdf 